\title{
LA MEJORA DEL APRENDIZAJE A TRAVÉS DE LAS NUEVAS TECNOLOGÍAS Y DE LA IMPLANTACIÓN DEL CURRÍCULO BIMODAL
}

\section{THE IMPROVEMENT OF LEARNING THROUGH NEW TECNOLOGIES AND THE IMPLEMENTATION OF BIMODAL CURRICULUM}

\author{
Esther Nieto Moreno de DiezMas \\ UNIVERSIDAD DE CASTILLA-LA MANCHA \\ Esther.Nieto@uclm.es \\ PERE MARQuès Graells \\ UNIVERSIDAD AUTÓNOMA DE BARCELONA \\ Pere.Marques@uab.ca
}

\begin{abstract}
RESUMEN
El contexto educativo, social y cultural actual considera el manejo de las nuevas tecnologías como una competencia clave o básica que todos los ciudadanos deben dominar para su pleno desarrollo personal, social y profesional y para el aprendizaje a lo largo de la vida. En este artículo presentamos diez claves para introducir las TIC en el aula de manera efectiva, y proponemos distintas estrategias y enfoques que abarcan desde el momento inicial de toma de contacto con las nuevas tecnologías hasta la inmersión en un modelo de aprendizaje basado en el currículo bimodal, que constituye en sí mismo una innovación de la enseñanza tradicional a través de las TIC y cuya ventaja fundamental es que cumple con el objetivo primordial de todo sistema educativo: mejorar la formación del alumno y promover el éxito escolar.
\end{abstract}

PALABRAS CLAVE

TIC, aprendizaje, currículo bimodal

\section{ABSTRACT}

The current educational, social and cultural context considers managing new technologies as a key or core competence that all citizens must master to their full personal, social and professional development and lifelong learning. In this paper we present ten keys to effectively introduce ICT in the classroom, and we propose different strategies and approaches ranging from the initial touchdown with new 
technologies to immersion in a learning model based on the bimodal curriculum, which constitutes itself an innovation of traditional education through ICT, and whose main advantage is that it meets the primary objective of any education system: improving learning and promoting school success.

KEY WORDS

ICT, learning, bimodal curriculum

\section{INTRODUCCIÓN: TIC, COMPETENCIA DIGITAL Y EDUCACIÓN}

Vivimos en un entorno en el que cada vez pasamos más tiempo y hacemos más cosas en un mundo que no es el mundo físico, sino el mundo virtual: el ciberespacio, y la educación no puede permanecer ajena a las profundas transformaciones que las tecnologías suponen, sino dar respuesta y preparar a los futuros ciudadanos para afrontar los nuevos retos, ya que el manejo de las tecnologías constituye una competencia básica para adaptarse al nuevo medio, al nuevo contexto, a las nuevas exigencias de la sociedad de la información y del conocimiento y solo los más adaptados a este nuevo panorama podrán mejorar sus posibilidades de éxito personales, sociales y profesionales.

Las TIC proporcionan a la educación poderosas herramientas, aplicaciones didácticas y no didácticas, informaciones e instrumentos de comunicación e interacción que pueden emplearse en el aula como ayuda o complemento para mejorar la motivación y los aprendizajes.

Pero el impacto de las tecnologías en la educación no se limita a proporcionar instrumentos para la mejora didáctica. La tecnología puede constituir en sí misma un elemento que desencadene y genere un cambio de los esquemas tradicionales por el que se viene abogando desde hace prácticamente un siglo. La introducción de las competencias básicas o clave en el sistema educativo supone uno de los últimos intentos por modernizar una educación anclada en la transmisión de conocimientos memorísticos. La enseñanza por competencias viene a poner de manifiesto la limitación de un sistema educativo basado en la memoria, puesto que lo importante no es lo que se sabe, sino qué se sabe hacer con lo que se sabe. La movilización de conocimientos, habilidades y valores para resolver problemas, para actuar, es 
más importante que su acumulación y en este sentido, las nuevas tecnologías posibilitan el almacenamiento casi ilimitado de información en "memorias externas" que alivian la necesidad de memorización y permiten desarrollar otras capacidades más útiles en este contexto tecnológico y además proporcionan un modelo pedagógico y de evaluación capaz de transformar los viejos esquemas tradicionales y mejorar los aprendizajes.

En este trabajo, se dan 10 claves para el uso de las TIC que servirán de guía al profesor que se inicie en su incorporación o que se dirija hacia la plena integración de las tecnologías en los procesos de enseñanza-aprendizaje a través del currículo bimodal. A lo largo de estas 10 ideas clave ofreceremos una visión realista de las ventajas e inconvenientes del uso de las TIC en el aula y consejos prácticos y concretos, ejemplos organizativos y descripción de actividades de aprendizaje y evaluación que contribuyan a mejorar la formación de los alumnos.

\section{DIEZ IDEAS PARA MEJORAR LOS APRENDIZAJES A TRAVÉS DE LAS TIC}

\subsection{USA LAS NUEVAS TEGNOLOGÍAS CUANDO APORTEN UN GRAN VALOR Ã̃ADIDO}

En primer lugar, al considerar integrar las TIC en la enseñanza y el aprendizaje hay que saber que introducir las TIC es más arriesgado que limitarse a emplear los materiales tradicionales: el libro de texto y la pizarra. Por ello, hay que valorar y conocer cuáles son las dificultades y los beneficios para el aprendizaje que conlleva su uso y utilizar las nuevas tecnologías cuando aporten un gran valor añadido.

El uso de las TIC en el aula puede conllevar, no solo inconvenientes de carácter técnico, como dificultades en la conexión a Internet, incompatibilidades entre dispositivos y archivos, desconfiguración del sistema operativo, averías en los equipos, rápida obsolescencia del hardware, etc., sino también problemas derivados de su uso pedagógico, tales como la escasez de contenidos educativos de calidad, la falta de tiempo del profesorado para buscar y crear nuevos recursos y para adquirir las competencias técnicas y funcionales necesarias, la dificultad de tener los dispositivos a punto al comenzar la clase, etc. A todo ello hay que sumar las eventuales distracciones en clase que puede conllevar el uso de las nuevas tecnologías, así como los peligros derivados de un mal uso de Internet, desde problemas de privacidad y 
difusión de datos o imágenes personales hasta el acceso a información inadecuada o la generación de adicciones tecnológicas. Todas estas eventualidades constituyen variables que es preciso prever o controlar cuando se pretenden incorporar las TIC a los procesos de enseñanza-aprendizaje.

La lista de posibles complicaciones parece larga. La cuestión es: ¿los inconvenientes del uso de las TIC compensan sus ventajas? Podemos avanzar una respuesta inicial a la cuestión. Más del $80 \%$ del profesorado encuestado en un estudio realizado por el grupo de investigación DIM desarrollado en la provincia de Burgos entre 2010 y 2014 considera que la preparación y gestión de actividades con las TIC supone un aumento significativo de trabajo, pero un porcentaje similar entiende que dicho esfuerzo merece la pena por las mejoras de los aprendizajes de los estudiantes (Marquès, 2014a).

Además, las investigaciones llevadas a cabo por el grupo de investigación DIM entre 2004 y 2010 (Marquès, 2014a) acerca del uso de distintas PDI o pizarras digitales interactivas (Dursi, Smart, Promethen y Mimio) revelan una visión muy positiva de su impacto en los procesos de enseñanzaaprendizaje. Según la mayoría del profesorado usuario (entre el 81\% y el 96\%, dependiendo del tipo de PDI), los mayores beneficios se detectan en el incremento de la motivación y participación del alumnado, que se califican como "altas" y en su mejor comprensión de los temas. El profesorado señala también, en un porcentaje bastante alto -y especialmente para las pizarras Smart y Promethean para las que oscila entre el $72 \%$ y el $84 \%$ - que las pizarras digitales mejoran su labor docente, facilitan la innovación metodológica, aumentan la satisfacción, motivación y autoestima docente y contribuyen a mejorar el aprendizaje de los estudiantes en general.

Asimismo, según el estudio acerca del impacto en los aprendizajes a través del uso de las tabletas digitales desarrollado por el grupo de investigación DIM en el periodo 2013-2014 (Marquès, 2014b), hay un acuerdo casi unánime del profesorado en que los beneficios más relevantes se cifran en el desarrollo del aprendizaje autónomo (97\%), alta motivación e implicación del alumnado $(97 \%)$, mejora de la comprensión en general $(95 \%)$, alto desarrollo de las competencias digitales $(95 \%)$, aumento de la creatividad (85\%), mejora de iniciativas y motivación para la investigación (85\%), mayor capacidad de memorización $(77 \%)$ y promoción de una continua actividad intelectual del alumno, debido a su interactividad y fácil acceso a distintos recursos (77\%).

Por otro lado, el estudio llevado a cabo por el grupo de investigación DIM en 2013-2014 (Marquès, 2014b) sobre el uso de las tabletas en educación especial, 
revela que las TIC no solo benefician los aprendizajes de los estudiantes más capaces, sino que mejoran en gran medida la motivación, el aprendizaje autónomo, las competencias digitales, la comprensión y el aprendizaje en general de alumnos con necesidades especiales, por lo que hay que considerar las TIC como una herramienta eficaz para el desarrollo de todo tipo de alumnado y especialmente para atender a la diversidad. Además, no solo los alumnos con necesidades especiales pueden verse beneficiados por el uso de las nuevas tecnologías, sino que estas también proporcionan distintas herramientas muy útiles para atender a los estudiantes con altas capacidades (Tárraga, Sanz-Cervera, Pastor y Fernández, 2014).

Un último aspecto que queremos comentar acerca de los diferentes estudios desarrollados por el grupo de investigación DIM y que será objeto de reflexión ulterior es la disociación entre el impacto que el profesorado otorga a la mejora del aprendizaje por parte de los alumnos, que es bastante alto, y a la mejora en el rendimiento académico, que es bastante limitada. Esta tendencia es especialmente llamativa en la investigación acerca de la introducción de la pizarra digital Dursi (Epson), ya que a pesar de que un $72 \%$ de los profesores consideran que los alumnos aprenden más, solo el $31 \%$ estima que se ha mejorado su rendimiento. Esta asimetría es recurrente en investigaciones posteriores. Así, por ejemplo, en el estudio acerca del impacto en los aprendizajes de las tabletas digitales (Marquès, 2014a) solo el 51\% del profesorado estima una mejora del rendimiento académico a pesar de que, paradójicamente, el $87 \%$ considera que el uso de tabletas contribuye a mejorar los aprendizajes de los alumnos en general.

\subsection{EL OBJETIVO NO HA DE SER INNOVAR, NI USAR LAS TIC, SINO MEJORAR LA FORMACIÓN DE LOS ALUMNOS Y CONSEGUIR UN MAYOR ÉXITO ESCOLAR}

El objetivo principal del sistema educativo es contribuir al éxito escolar y garantizar a los ciudadanos que puedan comprender e interactuar con el mundo que les rodea para vivir dignamente y desarrollar sus capacidades profesionales, personales y sociales. Sin embargo, España encabeza el fracaso escolar en Europa. Según Eurostat (2014), el 23,5\% de los estudiantes españoles abandonaron prematuramente el sistema educativo en el curso 2012-2013, mientras que la media comunitaria se sitúa en el $11,9 \%$ y el objetivo europeo 2020 para España es su reducción al 15\%. Por tanto, los esfuerzos del sistema educativo han de concentrarse en proporcionar más oportunidades y un contexto más favorable para fomentar el éxito escolar. 
Muchas veces, todo esto puede lograrse sin tecnología, pero otras, la tecnología es imprescindible.

Como decíamos, la educación ha de formar a los ciudadanos para desenvolverse en el mundo real, y una parte cada vez más importante de este mundo real es el "ciberespacio", donde cada vez pasamos más tiempo y hacemos más cosas que contribuyen a nuestro desarrollo personal, social y profesional, al aprendizaje y al aprendizaje a lo largo de la vida, con todos sus peligros y oportunidades.

Por ello, la adquisición de la competencia digital informacional ha de asegurarse a través de las distintas asignaturas del currículo de manera transversal, ya que sus beneficios son múltiples: colabora al aprendizaje de los contenidos propios de cada materia, brinda oportunidades metodológicas al profesor para atender a la diversidad y permite el desarrollo de estrategias, conocimientos y habilidades para desenvolverse en el mundo digital. La necesidad de dotar a los ciudadanos de herramientas para interactuar en este contexto justifica incluso la introducción de una asignatura específica, "Ciencias del Ciberespacio" que les formaría en habilidades de búsqueda, tratamiento, creación y comunicación de información en el ciberespacio y fomentaría el uso crítico de las TIC.

Las TIC han de emplearse, por tanto, como instrumento para mejorar la formación del estudiante y combatir el fracaso, para dotarle de un bagaje útil a lo largo de su vida y contribuir a la mejora de los procesos de enseñanzaaprendizaje, aumentando la motivación y la participación del alumnado y proporcionando al profesor herramientas para atender a la diversidad. Además, cuando se trata de atender al alumnado con necesidades especiales, las TIC proporcionan aplicaciones que operan cambios significativos en la autonomía, la comunicación, y la estimulación cognitiva, a través de sintetizadores de voz, adaptadores, sistemas de reconocimiento de voz y aplicaciones y sistemas alternativos y aumentativos de la comunicación (SAAC), entre otros.

\subsection{LA ERA INTERNET SUPONE UN CAMBIO DE CONTEXTO QUE SE TRADUCE EN LA NECESIDAD DE UN CAMBIO EN EL PARADIGMA EDUCATIVO Y EN LOS OBJETIVOS DE LA EDUCACIÓN}

La educación tradicional parte de un enfoque centrado en el profesor que emplea una metodología basada en la transmisión de unos conocimientos y de 
unos saberes estereotipados que el alumno debe memorizar y repetir. Los aprendizajes se inscriben en una concepción "bancaria" del aprendizaje y alienante del individuo (Freire, 2008) que consiste en el depósito y acumulación de conocimientos sin cuestionarlos ni elaborarlos, para repetirlos y reproducirlos. Este esquema educativo, aún presente en las aulas, ha sido cuestionado desde las aportaciones de distintas corrientes psicológicas y pedagógicas como el constructivismo, la escuela nueva, el aprendizaje dialógico o las inteligencias múltiples que colocan al aprendiz en el centro del proceso de enseñanza-aprendizaje y acentúan la importancia de su participación activa y experiencial para la construcción e integración de los aprendizajes con sentido crítico y dándole oportunidades para que aprenda según su ritmo, estilo y talentos. Además, el énfasis que la enseñanza tradicional ponía en los contenidos y la memorización se sustituye por el concepto de competencia. Las competencias básicas son transferibles, multidimensionales, transversales e inclusivas (Nieto, 2010, 85-86), de manera que abogan por una enseñanza centrada en la adquisición integrada de conocimientos, valores y destrezas no rutinarias que puedan movilizarse para actuar de manera relevante y resolver problemas y que puedan transferirse de manera creativa a distintos contextos y situaciones.

Si la enseñanza tradicional ha sido puesta en cuestión desde distintas corrientes pedagógicas o psicológicas, la revolución de Internet ha transformado nuestra sociedad en un espacio dinámico, global e intercomunicado, en el que el cambio constituye un elemento central (Majó y Marquès, 2001) y cuyos retos ya no pueden ser afrontados con los viejos esquemas educativos. Internet profundiza la brecha entre la educación y lo que se demanda de ella, que es atender a las verdaderas necesidades de sus usuarios que han de saber actuar en el mundo que los rodea que incluye, no solo el espacio físico o natural, sino también el ciberespacio. Además, se hace urgente la necesidad de "aportar herramientas que permitan transformar la información en conocimiento" (Escamilla, 2009,146), en un contexto en el que el acceso a una gran cantidad de información se encuentra limitado y mediatizado por las propias capacidades de selección, comprensión, tratamiento y valoración crítica.

Incluso la propia escuela se pone en cuestión como elemento centralizador y validador de conocimientos, de manera que gracias a la ingente cantidad de contenidos y posibilidades que proporciona la red y a la facilidad de su acceso, "el aprendizaje puede suceder en cualquier lugar" (Attwell, 2013, 190). 
En este sentido, las nuevas tecnologías ofrecen entornos personales de aprendizaje (EPA) llenos de posibilidades que el sistema educativo debe aprovechar. La educación debe garantizar a todos el empoderamiento que supone el uso de las nuevas tecnologías de manera eficaz en distintos contextos (Marquès, 2013a) para articular el aprendizaje a lo largo de la vida y para la configuración de los propios EPA, para lo cual, es necesario que las TIC se usen frecuentemente y "que sirvan para enriquecer la manera en la que aprenden las personas tanto de forma individual como con otros" (Castañeda y Adell, 2013,15).

La posibilidad de estar siempre conectados a Internet (a través de $i$-phones o smartphones, tabletas y ordenadores) nos permite llevar a cabo actividades necesarias para nuestro desarrollo profesional, académico, personal y social. Internet nos proporciona un acceso permanente a una gran cantidad de información, así como la posibilidad de compartirla, reelaborarla y comunicarla. Esta situación puede considerarse como un salto evolutivo que convierte al Homo sapiens en "i-person", permanentemente conectada a Internet y preparada para interactuar en un contexto cultural dominado por las nuevas tecnologías (Marquès 2011).

En este contexto, Internet supone la adición de un nuevo lóbulo a nuestro cerebro, el "lóbulo Internet" (Marquès, 2013b, 66), de manera que la capacidad física humana de almacenamiento de datos ya no se limita, en este caso, al cerebro, a la memoria interna, sino que se encuentra ampliada por una poderosa memoria externa constituida por los recursos accesibles a través de Internet en forma de blogs, páginas web o redes sociales y apuntes o investigaciones propias en la nube, en pendrives o discos duros, que pueden ser recuperados con facilidad y rapidez a golpe de "clic".

Por todo ello, es necesario que la educación se haga eco de los cambios contextuales y culturales que están convirtiendo nuestra sociedad en un espacio muy tecno-dependiente, global, complejo y en continua transformación. Estos cambios han de afectar a todos los elementos del currículo: los objetivos y contenidos han de hacer especial hincapié en la adquisición de competencias y especialmente de aquellas que favorecen el aprendizaje continuo y los materiales y recursos han de integrar las nuevas herramientas TIC (pizarra digital, aula 2.0, plataforma educativa EVA, Internet...), lo que afecta también a las infraestructuras, que requieren de una nueva organización. 
Además, la metodología tradicional ha de adaptarse a los nuevos sistemas de trabajo y optar por el desarrollo de proyectos, tareas colaborativas y resolución de problemas de manera individual y grupal. En este contexto, la evaluación no puede centrarse en el aprendizaje memorístico propio de la enseñanza tradicional, sino adaptarse a un nuevo modelo de enseñanza basado en la adquisición de habilidades y capacidades y ser coherente con los nuevos sistemas de aprendizaje llevados a cabo en el aula a través de las TIC.

Por tanto, solo si se produce la integración de las TIC en todos los elementos del currículo se podrá elaborar un modelo consistente y coherente del aprendizaje en el marco de la era Internet. Para que el docente pueda llevar a cabo las transformaciones requeridas es necesario que, por un lado, sepa integrar la pedagogía en el contexto concreto de la asignatura a impartir y, por otro, que disponga de unos conocimientos TIC también contextualizados tanto en la pedagogía, como en la asignatura. Este es el esquema por el que aboga el modelo TPACK (Mishra y Koehler, 2006) que explica cómo el docente ha de imbricar e integrar de manera interactiva tres tipos de conocimientos para poder diseñar secuencias de aprendizaje: conocimientos pedagógicos, conocimientos disciplinares y conocimientos tecnológicos, de manera que manejar la aplicación de las TIC en la asignatura que el docente imparte se convierte en un elemento tan importante como dominar sus contenidos curriculares y su pedagogía.

\subsection{LAS COMPETENCIAS QUE Había QUE ADQUIRIR EN EL PASADO HAN CAMBIADO}

Antes, la escuela tenía que enseñar a leer y escribir, a hacer "a mano" todo tipo de operaciones y a memorizar todo lo que se necesitaba saber. Hoy, sigue siendo necesario que aprendamos a leer y escribir, pero en más soportes y formatos y no es necesario que sepamos hacer todo tipo de operaciones "a mano", sino que sepamos manejar la calculadora para ello. Además, más que memorizar conceptos, lo que es importante es que sepamos dónde localizar la información que necesitamos. Por otro lado, el sistema educativo tiene que asegurar la adquisición de determinadas competencias (las competencias básicas o clave), entre las que se encuentra la competencia digital.

La competencia digital ocupa un lugar instrumental entre el resto de las competencias, ya que posibilita el acceso a conocimientos multidisciplinares, y su dominio en la era Internet es necesario para el desarrollo integral, social, personal y profesional de los ciudadanos. Además, a diferencia de otras 
competencias, la competencia digital solo se puede adquirir trabajando con dispositivos digitales, pero como el resto de competencias ha de ser trabajada de manera interdisciplinar, es decir, desde todas las materias del currículo. En síntesis, el desglose de competencias básicas que se han de trabajar a lo largo de la escolarización obligatoria, se especifica en la tabla 1.

\section{INSTRUMENTOSYAPLICACIONES}

1. Seleccionar, configurar, programar y usar dispositivos digitales según las tareas.

2. Utilizar aplicaciones de edición de textos, presentaciones multimedia, tratamiento de datos numéricos.

3. Utilizar aplicaciones de edición de imagen fija, sonido e imagen en movimiento. TRATAMIENTO DE LA INFORMACIÓN Y ORGANIZAR ENTORNOS DE TRABAJO Y APRENDIZAJE
4. Buscar, contrastar y seleccionar información digital de diversas fuentes.
5. Construir nuevo conocimiento personal mediante estrategias de tratamiento de la información con el soporte de aplicaciones digitales.
6. Organizar y utilizar un entorno digital personal de trabajo y aprendizaje.

\section{COMUNICACIÓN INTERPERSONALY COLABORACIÓN}

7. Usar entornos de comunicación interpersonal y publicación virtual para compartir información

8. Hacer actividades en grupo usando herramientas y entornos virtuales de trabajo colaborativo HÁBITOS, CIVISMO E IDENTIDAD DIGITAL

9. Realizar acciones de ciudadanía y desarrollo personal utilizando los recursos digitales. 10. Fomentar hábitos de uso saludable de las TIC para prevenir riesgos

11. Actuar de forma critica y responsable en el uso de las TIC, considerando aspectos éticos, legales, de seguridad, de sostenibilidad y de identidad digital.

Tabla 1. Pere Marquès, Competencias básicas del ámbito digital, 2014 c.

\subsection{ES IMPORTANTE DISTINGUIR LAS TIC IMPRESCINDIBLES DE LOS "GADGETS" OPCIONALES}

La tecnología nos puede deslumbrar y nos puede hacer perder mucho tiempo y dinero; por eso es necesario reflexionar y valorar qué herramientas son imprescindibles y cuáles son accesorias y superfluas.

En un aula TIC o aula 2.0, se entiende que ha de haber una pizarra digital interactiva o un vídeo-proyector, un ordenador fijo de aula conectado a la pizarra digital interactiva o vídeo-proyector, un ordenador por cada alumno y conexión a Internet para todos los puestos de trabajo. Por otro lado, se precisa de una intranet o plataforma educativa de centro que proporcione a profesores y alumnos correo electrónico, un espacio de portafolios digital, foros, etc., un portal de centro y una biblioteca de recursos didácticos de 
centro. En cuanto al software, es necesario contar con editor gráfico, de textos, de sonido, vídeo y multimedia; calculadora, editor de mapas conceptuales (como cmaptools), líneas de tiempo (DIPITY), etc. Si es posible, también es útil contar con un lector de documentos y una impresora de aula.

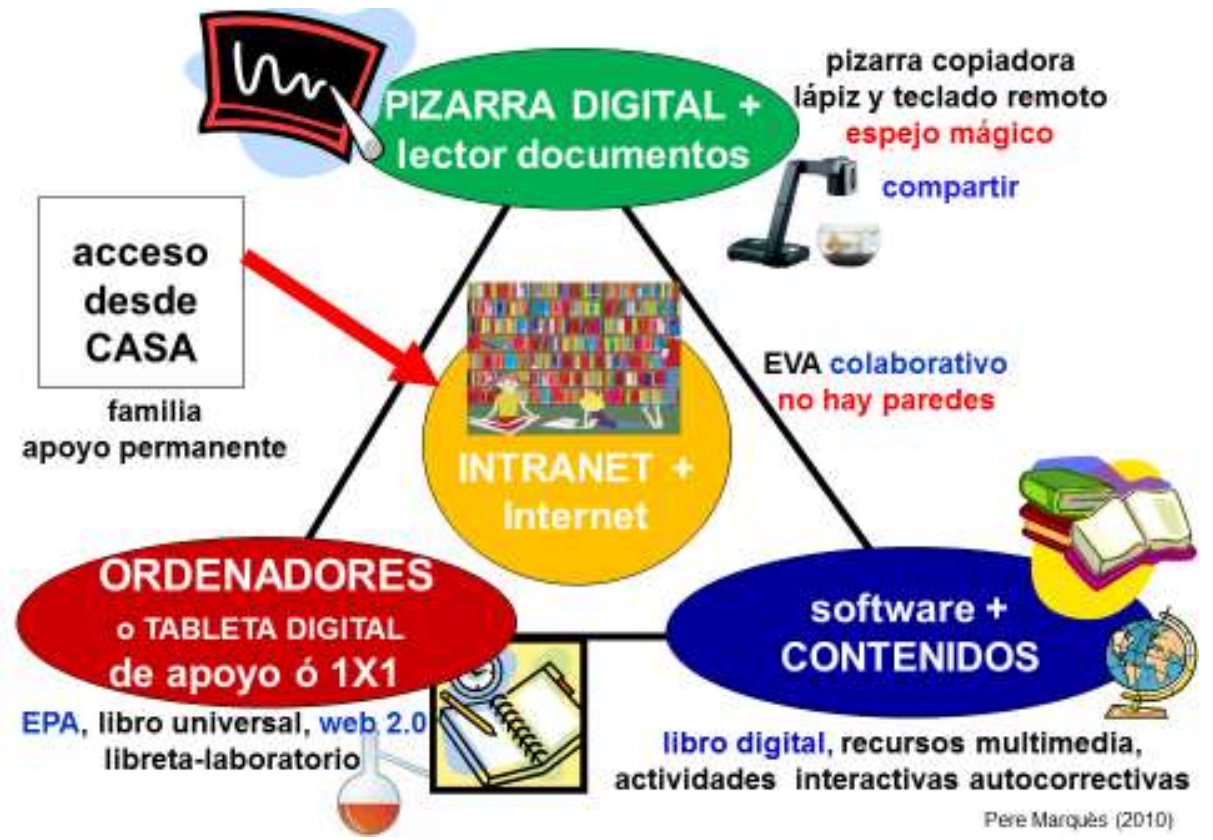

Gráfico 1. Pere Marquès, Recursos del aula 2.0, 2014c.

Las tabletas pueden ser muy útiles como alternativa a los netbooks en un aula con pizarra digital y conexión a Internet. En este sentido existen varios modelos organizativos. Se puede crear un aula específica, en la que se encuentran las tabletas digitales, a la que deben trasladarse los alumnos. Esta opción no es la mejor, debido a la incomodidad que suponen los traslados. Una alternativa que favorece la familiarización con estos dispositivos en el aula es la existencia de algunas tabletas fijas de apoyo en la clase que pueden emplearse en diversos trabajos individuales o grupales. Este modelo puede complementarse con los carros de tabletas digitales. El centro puede disponer de carros, en los que las tabletas puedan transportarse, almacenarse y cargarse, dispuestos para que el profesor los traslade al aula cuando lo requiera. No obstante, es muy conveniente, especialmente a partir de los últimos cursos de primaria, que los alumnos dispongan de una tableta o netbook, ya sea propiedad del centro o de cada alumno (opción BYOD: "bring your own device").

Muchas son las utilidades de las tabletas y netbooks. Sus aportaciones se pueden clasificar en cuatro aspectos: el acceso a la información, el acceso a 
aplicaciones de materiales curriculares, la ampliación de posibilidades para procesar los datos y, por último, el acceso a herramientas para comunicarse.

Así, en lo que respecta al primer aspecto, las tabletas nos permiten acceder a la información, visualizarla y almacenarla, localmente o a través de la nube. Sirven como fuente de información a la que podemos acceder a través de navegadores, buscadores, códigos QR, enciclopedias, mapas, geolocalización, programas de radio o televisión, realidad aumentada, etc. y nos permiten la captura digital del mundo físico mediante la cámara, el micrófono, aplicaciones para grabar voz y sonido, como ASR, etc.

En segundo lugar, a través de la tableta, podemos acceder también a materiales curriculares, como libros digitales, ejercicios multimedia autocorrectivos, simuladores, webquests y juegos que pueden ser muy útiles en el proceso de enseñanza-aprendizaje. Además, ofrecen nuevas posibilidades de atender a la diversidad a través de actividades personalizadas (de refuerzo, ampliación, prácticas y memorísticas) y también impactan positivamente en la configuración de entornos virtuales de aprendizaje (EVA).

En tercer lugar, la tableta constituye un excelente instrumento para procesar los datos, organizarlos, transformarlos, aplicarlos y crearlos, mediante herramientas como las hojas de cálculo, traductores, reconocimiento óptico de caracteres (OCR), editores de texto, notas, mapas conceptuales, líneas del tiempo, multimedia, agenda, etc.

En cuanto a la comunicación, las tabletas favorecen la comunicación interpersonal, mediante el correo electrónico o vídeo-comunicaciones y la comunicación social, a través de la creación o incorporación a grupos (foros, redes...) o publicaciones on-line (blogs, wikis), entre otros.

En definitiva, muchas son las ventajas que comportan las tabletas y netbooks en su uso educativo y cuando se usan con una buena metodología, unas buenas actividades y una evaluación coherente consiguen aumentar la motivación e implicación del alumnado, mejoran sus aprendizajes y competencias y logran una mayor eficacia didáctica. 


\subsection{Tener PIZARras o tabletas digitales No ASEgura QUE LOS ALUMNOS APRENDAN MÁS}

El mero uso de artefactos digitales no implica directamente una mejora de los procesos de enseñanza aprendizaje; la clave esencial del éxito es realizar buenas actividades didácticas. Es, por tanto, necesario distinguir tecnología y metodología. La tecnología, a través de los distintos dispositivos digitales constituyen una herramienta que por sí sola no garantiza los aprendizajes. El aprendizaje se produce cuando el docente emplea consciente y eficazmente las TIC a través de una metodología adecuada aplicada al aprendizaje disciplinar y competencial (TPACK). Por tanto, para que las TIC tengan un valor pedagógico es necesario contar con un enfoque metodológico que se centre en proporcionar a los alumnos experiencias que estimulen sus actividades mentales y el desarrollo de sus procesos cognitivos de orden inferior y superior.

Así pues, si utilizamos las TIC para hacer lo mismo que antes, su impacto positivo en los aprendizajes es limitado. Pongamos como ejemplo la pizarra digital. Si el profesor explica de manera magistral a través de diapositivas proyectadas en ella, no se produce ningún cambio metodológico, sino que la herramienta tecnológica, en este caso, la pizarra digital sustituye a otra anterior, la pizarra tradicional. Por tanto, se produce una sustitución que no comporta prácticamente ningún cambio funcional (Puentedura, 2014). Para extraer de las TIC el máximo rendimiento en los procesos de enseñanzaaprendizaje y en el desarrollo de procesos cognitivos y competencias de los estudiantes, es necesario que se opere una transformación y redefinición metodológica de las tareas y actividades de aprendizaje a través de la tecnología, lo que nos permitirá dar un salto cualitativo, mejorar los aprendizajes y preparar a los futuros ciudadanos para interactuar en una sociedad dominada por las TIC. Así por ejemplo, en vez de usar la pizarra digital para seguir dando clase de la misma manera, puede emplearse para que los alumnos presenten los resultados de proyectos colaborativos realizados a partir de la reelaboración y síntesis de informaciones y recursos en diversos formatos recopilados de Internet, de materiales digitales creados por ellos mismos y de sus propias reflexiones críticas. De esta manera, los alumnos comprenden, aplican, analizan, evalúan y crean contenidos integrando distintas tecnologías y son capaces de presentar lo aprendido en sus proyectos de trabajo de manera multimodal empleando todo tipo de materiales audiovisuales. 
Multiárea. Revista de didáctica Núm. 7 (2015): 7-30

ISSN: 2444-9431
Esther Nieto Moreno de Diezmas / Pere Marquès Graells La mejora del aprendizaje a través de las nuevas tecnologías

Por tanto, el tipo de tareas y actividades que se realizan en el aula con las TIC tienen un papel fundamental en el cambio metodólogico necesario para mejorar los aprendizajes. En la tabla 2 se enumeran una serie de actividades didácticas, para realizar con tabletas o netbooks que pueden operar un cambio significativo en el contexto del aula.

- Estudiar y realizar ejercicios utilizando:

- Apps: grafomotricidad, pre-lectura, dibujo, ortografía, sintaxis, cálculo, listening, traducción, simuladores, Google Earth... y libros de texto

- Contenidos educativos on-line elaborados para los netbooks

- Contenidos educativos que prepare cada profesor en su blog docente o en la plataforma educativa del centro

- Alumnos buscadores de recursos sobre temas de la asignatura, que luego explican a sus compañeros en la PD.

- Alumnos profesores que preparan una presentación multimedia sobre un tema para exponerlo en clase en la PD

- Alumnos investigadores que afrontarán proyectos, problemas complejos, webquest... y luego presentarán a la clase en la PD.

- Trabajos colaborativos de toda la clase a lo largo del curso:

- Wiki glosario de asignatura y wikis temàticas de alumnos especialistas

- Periódico digital (imprimible) con diversas secciones

- Blog diario de la asignatura: cada día dos alumnos hacen una sintesis

Alumnos creadores y periodistas, elaborarán un material tipo:

- Fichas o montajes multimedia de noticias a partir de fotos

- Entrevistas de audio o vídeo a personas del barrio, reportajes...

- Cuentos, poemas y narraciones multimedia (en español, en inglés...)

- Montajes musicales: canciones en inglés, componer música...

- Vídeos tutoriales que expliquen cómo hacer una cosa determinada.

-Materiales didácticos multimedia sobre temas de clase

- Actividades individuales cuando cada alumno tiene una tableta:

- Blog personal de cada alumno.

- Toma de notas y elaboración de apuntes (memoria auxiliar)

- Cada alumno hace "su" libro de la asignatura.

- Actividades en la red social de la clase

Realizar exámenes con apuntes y acceso a internet. Como se sugiere en la metodología del "currículum bimodal"

- En Educación Especial : lectores de documentos y OCR que tengan la función de lupa o de lectura de voz del documento, aplicaciones de reconocimiento de voz...

Pere Marquàs (2012)

Tabla 2. Pere Marquès, Modelos de actividades didácticas con tabletas y netbooks, 2014c. 


\subsection{NO BASTA CON PROPONER A LOS ALUMNOS BUENAS ACTIVIDADES DE APRENDIZAJE}

Tampoco basta con diseñar buenas actividades de aprendizaje; es necesario gestionarlas bien e introducirlas en el momento adecuado con eficacia y eficiencia. Para ello, hay que tener en cuenta aspectos didácticos y organizativos y cuestiones como la gestión del tiempo en el aula de las distintas actividades de enseñanza aprendizaje. El primer año que se introducen las TIC en el aula es conveniente que el uso del ordenador, tableta o netbook no exceda del $30 \%$ del tiempo de clases, para poder ir regulando el proceso a partir de la experiencia. Poco a poco, el ordenador puede ir sustituyendo al libro como fuente de información y al cuaderno, como lugar de trabajo, prácticas, ejercicios, compilación de apuntes, etc. Además, se abren nuevas posibilidades para comunicarse, compartir, debatir, crear y trabajar colaborativamente.

Una sugerencia en la organización de los tiempos de uso en el aula de secundaria, sería la reducción temporal tanto de las explicaciones del profesor, como del trabajo individual o grupal sin TIC, de manera que se dedicaría un $20 \%$ del tiempo disponible a cada una de estas metodologías, mientras que el $60 \%$ del tiempo restante podría dedicarse al trabajo individual o grupal de los alumnos con el ordenador, netbook o tableta $(30 \%)$ y a exponer los resultados de sus proyectos o investigaciones $(30 \%)$. Esta propuesta de organización temporal en el aula se especifica en la tabla 3.

\begin{tabular}{|c|c|c|c|}
\hline $\begin{array}{c}20 \% \\
\text { explicaciones } \\
\text { del profesor } \\
\text { con PD }\end{array}$ & $\begin{array}{c}30 \% \\
\text { exposiciones } \\
\text { trabajo alumnos } \\
\text { con PD }\end{array}$ & $\begin{array}{c}30 \% \\
\text { trabajo } \\
\text { individual/grupal } \\
\text { con PC }\end{array}$ & $\begin{array}{c}20 \% \\
\text { trabajo } \\
\text { individual/grup } \\
\text { al sin PC }\end{array}$ \\
\hline
\end{tabular}

Tabla 3. Pere Marquès, Organización temporal con TIC para secundaria, 2014c.

Para introducir las TIC en el aula de manera exitosa hay que prever contingencias como la avería de los aparatos o problemas con Internet y preparar actividades alternativas de manera que tales eventualidades no determinen que el proceso de enseñanza se lleve a cabo. También es importante plantearse cuáles son las ventajas e inconvenientes de los distintos modelos funcionales de introducción de ordenadores en el aula. Pueden usarse como soporte del libro de texto digital y para hacer ejercicios autocorrectivos, pero en este caso, se trata de una simple sustitución del libro de texto por el digital, de manera que se sobrecarga el trabajo docente y el aprendizaje sigue fundamentándose en el aspecto memorístico. Otra opción es 
que el ordenador o tableta se emplee para acceder a la plataforma educativa del centro donde el profesor habrá organizado recursos para el estudio, ejercicios, foros, etc. Además de los inconvenientes del modelo anterior, se añade el riesgo de exceso de planificación. La tercera opción es utilizar el ordenador como instrumento multifuncional para acceder a la información, el proceso de datos y la comunicación para usar de forma autónoma en actividades prácticas tipo proyecto (a menudo colaborativas y con apoyo de recursos de la web 2.0). Este modelo proporciona experiencias de aprendizaje más ricas, aunque se corre el riesgo de dispersión del alumno.

En definitiva, para sacar el mayor rendimiento a las TIC hay que considerar los siguientes aspectos:

- Promover el uso del lenguaje audiovisual (fotos, dibujos...) en los trabajos.

- Buscar la participación de los estudiantes a través de roles: tutores aventajados que ayudan a los compañeros; especialistas temáticos que asesoran a los demás en "su" tema; monitores TIC que ayudan al profesor...

- Buscar la construcción personal del conocimiento mediante actividades aplicativas contextualizadas que impliquen seleccionar y analizar, organizar y relacionar, interpretar y aplicar información.

- Desarrollar la curiosidad, iniciativa, autonomía, autorregulación, responsabilidad y capacidad de superación de los alumnos.

- Priorizar la reflexión y el pensamiento crítico, la autocrítica y la argumentación, la imaginación y la creatividad, frente al memorismo.

- Trabajar las competencias básicas y las TIC con actividades de descubrimiento, investigación y aplicación de conocimientos.

- Utilizar múltiples metodologías (clase inversa...) y recursos variados.

- Atender la diversidad y la interculturalidad.

\subsection{Para Que El Rendimiento escolar Mejore no basta Con GRear BUENAS ACTIVIDADES Y GESTIONARLAS ADECUADAMENTE}

Ya veíamos al principio cómo en los estudios del grupo de investigación DIM, las opiniones del profesorado revelaban una falta de correspondencia entre el progreso en el aprendizaje de los alumnos que utilizan las TIC y la mejora de su rendimiento académico. Estos resultados están en la línea de otras investigaciones nacionales e internacionales que no consiguen establecer una relación causal entre el uso de ordenadores y la mejora del rendimiento. Así, Fuch y Woessman (2004) encuentran mejora cuando el uso de las TIC es 
esporádico, pero con un uso más continuado, los resultados académicos incluso empeoran. Machín, McNally y Silva (2007) aportan resultados no concluyentes, ya que registran un efecto positivo en el uso de las TIC en lectura y en ciencias, pero no en matemáticas. Banerjee, Cole, Duflo, y Linden (2007) sí que registran mejores resultados en matemáticas cuando se trabaja en el aula con TIC, pero Angrist y Lavy (2002) detectan que las notas no solo no mejoran, sino que descienden cuando se usan los ordenadores para enseñar matemáticas. Algo más positivo es el estudio de Cabras y Tena (2013) que utilizan la base de datos de PISA y comparan los resultados de los alumnos que usan ordenadores, tabletas o netbooks en el centro educativo con los que no tienen acceso a dichos dispositivos. La investigación revela que aquellos alumnos que usan ordenadores en el aula consiguen mejores resultados en la prueba de matemáticas, pero las diferencias no son significativas.

Esta ausencia de conexión entre las TIC y la mejora de las notas, y sobre todo, el hecho de que en las investigaciones del grupo DIM, los profesores reconozcan que los alumnos aprenden más con las TIC pero no sacan mejores resultados sugiere la existencia de lo que podría denominarse "la paradoja del uso educativo de las TIC", que consiste en el hecho de que los profesores y los propios alumnos consideran que utilizando las TIC se aprende más, pero no se consigue un mayor rendimiento académico.

La causa de este hecho es que, muchas veces, la introducción de las TIC en el aula supone una transformación de los métodos de trabajo y aprendizaje, pero estos cambios no llegan a afectar a la evaluación, que sigue basándose en aprendizajes memorísticos, y como el uso habitual de las TIC no mejora la memoria, el resultado es que los resultados académicos no mejoran.

Por tanto, para que la paradoja desaparezca es necesario cambiar de paradigma. Las TIC constituyen el eje de un cambio metodológico que ha de penetrar en todos los elementos del currículo, hasta llegar a la evaluación. Para ello, es necesario relativizar la importancia de la memorización en la era Internet en la que la $i$-person puede acceder de manera inmediata a datos e informaciones que amplían de manera extraordinaria los límites de su memoria y hacer más hincapié en la adquisición de estrategias, habilidades y competencias que permitan a los futuros ciudadanos desarrollarse plenamente y responder a los retos de un contexto social y cultural cambiante, globalizado e intercomunicado. Así pues, la excesiva memorización propia de la educación tradicional deja de tener sentido, dado que tenemos la información siempre al alcance, y cobra importancia en su lugar la capacidad para comprenderla, procesarla, analizarla, transformarla y evaluarla con sentido crítico. 
Estos son los principios del currículo bimodal (Marquès, 2013a) que aboga por un modelo que no solo contemple la realización de actividades en el aula con las TIC, sino que se realice una evaluación adecuada y coherente con los aprendizajes adquiridos.

El currículum bimodal se compone de dos aspectos que afectan a la metodología y la evaluación: "saber hacer" y "memorización del vocabulario básico" (Gráfico 2).

Así pues, por un lado, los alumnos han de aprender a hacer, es decir, adquirir competencias, habilidades, conocimientos y valores transferibles a distintos contextos para resolver problemas, desde el punto de vista de la acción. Este aspecto se trabaja a través de una buena variedad y cantidad de actividades prácticas, ejercicios y proyectos individuales y colaborativos que consistan en investigar, aplicar, valorar y crear para cuyo desarrollo los alumnos tengan que adoptar distintos roles: profesores, periodistas, ayudantes, investigadores, etc. Además, se enseñará al alumno a que elabore sus "apuntes personales" que contendrán resúmenes, esquemas, fórmulas, ejemplos... El alumno puede usar estos "apuntes" como memoria auxiliar en la realización de actividades de clase y también en las pruebas prácticas de evaluación. Además se ha de potenciar la evaluación continua y generar contextos de reflexión sistemática a partir de la participación del alumno tanto en la valoración de las producciones de los compañeros (coevaluación), como en la evaluación de sus propias realizaciones (autoevaluación), de manera que aprenda a regular sus propios aprendizajes y a desarrollar más autonomía a la hora de tomar decisiones acerca de cómo seguir aprendiendo.

El segundo aspecto que se ha de trabajar es la adquisición de un vocabulario básico que el alumno debe manejar, conocer y recordar que sirve como base para desarrollar sus competencias y realizar actividades prácticas. La metodología empleada para la adquisición de estos conceptos clave es la de la memorización reconstructiva, de manera que en vez de recurrir a la memorización mecánica, se parte de la comprensión e interiorización del vocabulario y, mediante un proceso de andamiaje se enseña a los estudiantes a construir sus propias definiciones. El profesor selecciona el vocabulario imprescindible de cada lección y el alumno elabora su glosario personal. Para comprobar el dominio del vocabulario por parte del alumno se realizarán exámenes teóricos sobre el glosario, de manera que el 70\% de las preguntas de estos exámenes teóricos versarán sobre los términos contenidos en él. 
Todo el proceso se completa con una buena acción tutorial que sea capaz de detectar precozmente las dificultades y colaborar en el desarrollo integral del alumno, haciendo especial hincapié en las emociones, la motivación y la autoestima y que reconozca y atienda las diferentes inteligencias y talentos de los estudiantes.

\section{CURRICULUM BIMODAL: 2 tipos de actividades}

\section{Bimodalidad metodológica y en la evaluación}

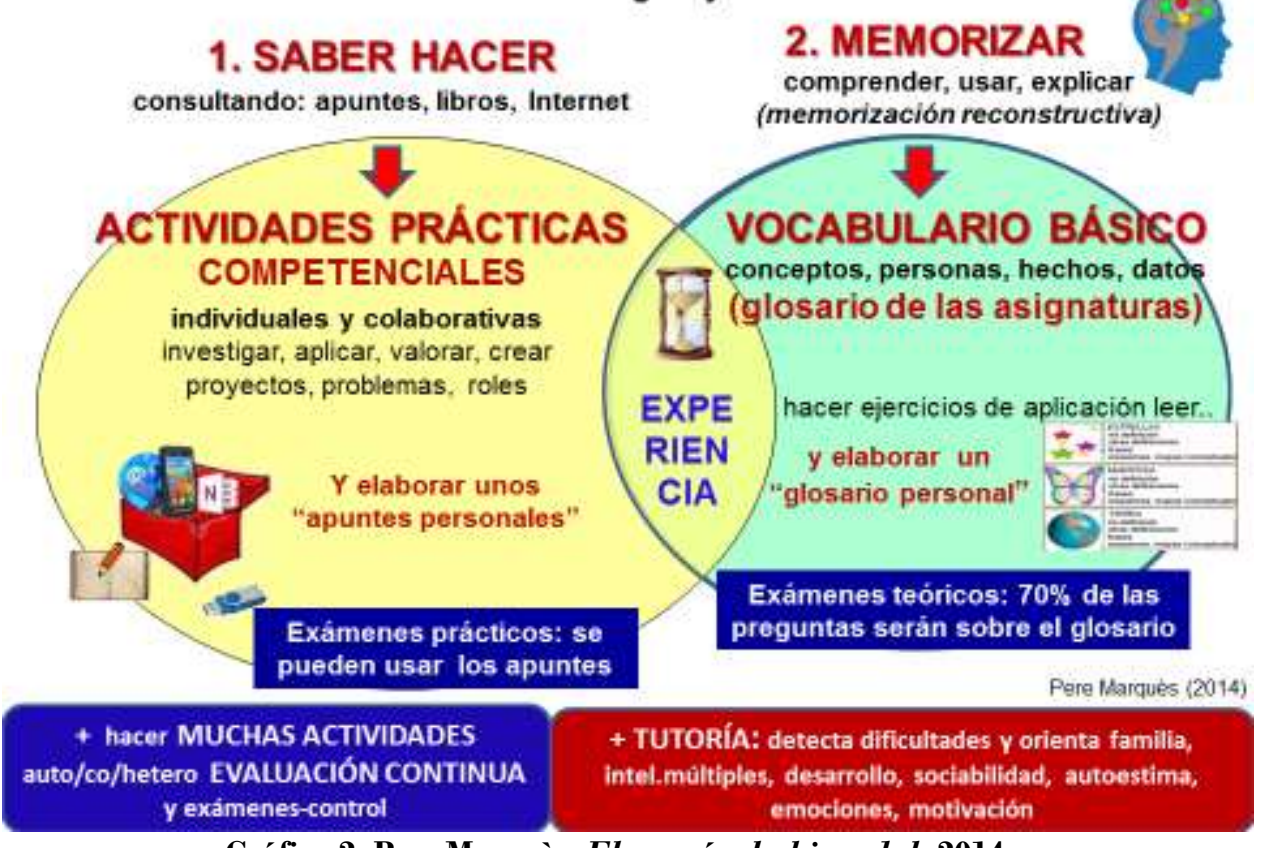

Gráfico 2. Pere Marquès, El currículo bimodal, $2014 c$.

En definitiva, el currículo bimodal se basa en cuatro principios: elaboración de apuntes por parte de los alumnos, realización de exámenes prácticos con apuntes, fabricación de un glosario básico personal y exámenes teóricos sobre el glosario.

En la investigación del grupo DIM realizada en 13 centros piloto de primaria y secundaria el curso 2013-2014, se detecta que cuando se trabaja el currículo bimodal y se aplican estos cuatro principios básicos, no solo mejoran los aprendizajes, sino que también mejoran las calificaciones académicas (gráfico 3). Además, el efecto más relevante del currículo bimodal es su capacidad para combatir el fracaso, puesto que con su aplicación aumenta considerablemente el porcentaje de alumnos que aprueban. 


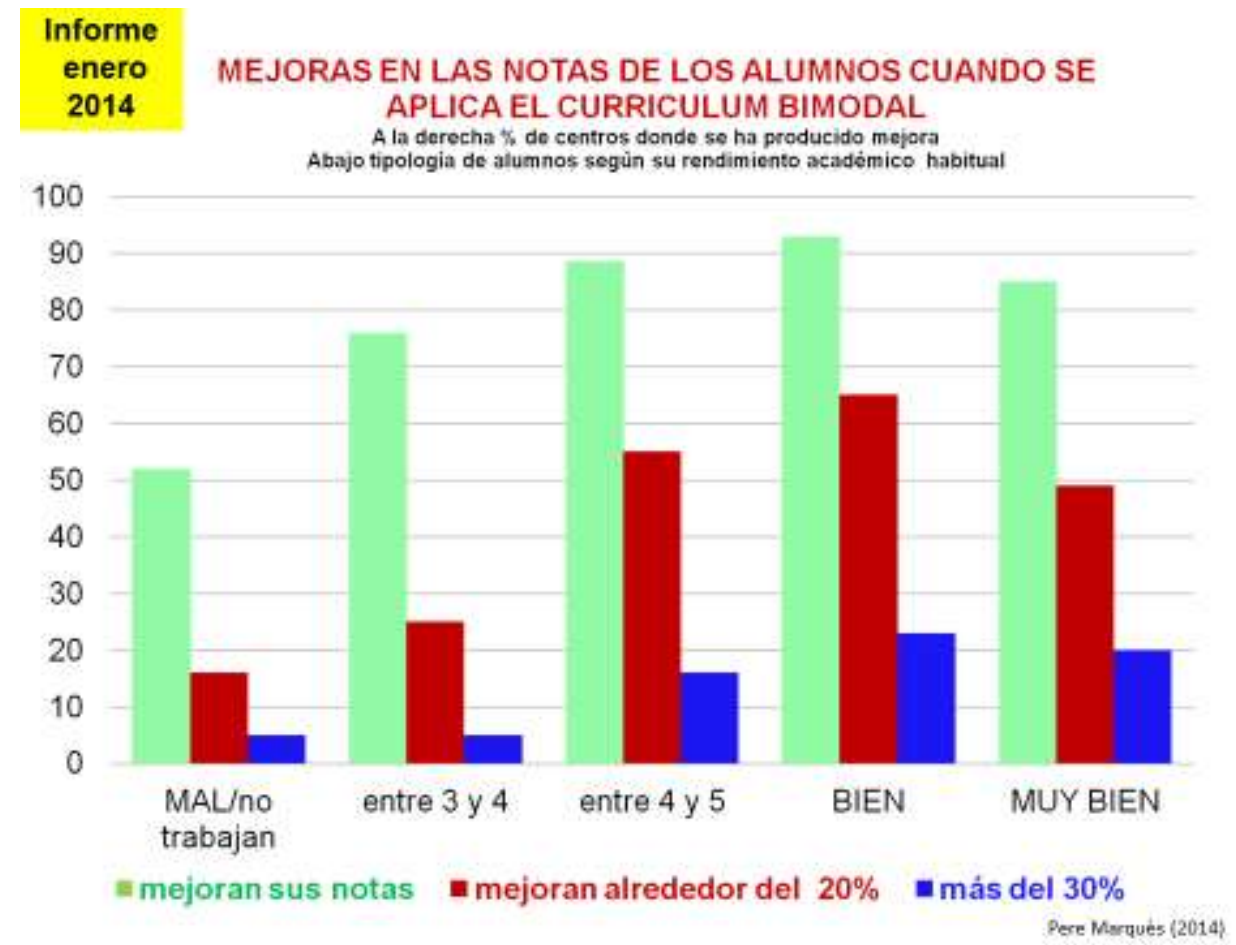

Gráfico 3. Pere Marquès, Mejoras en las notas de los alumnos con el currículo bimodal, 2014c.

\subsection{No BASTA CON TENER RECURSOS. SE REQUIERE FORMACIÓN Y COMPROMISO}

Si no hay compromiso por parte del docente o el profesor no cuenta con la formación adecuada, la experiencia puede fracasar. Para que el cambio metodológico a través de las TIC pueda llevarse a cabo, el profesor ha de "poder", "saber" y "querer". Así pues, es imprescindible que existan recursos que posibiliten el trabajo con las nuevas tecnologías, pero no es menos necesario que el profesor esté formado tanto en el uso instrumental como didáctico de las TIC, que sepa cómo incorporarlas a su práctica docente y que se comprometa a hacerlo, que quiera y esté dispuesto a hacer el esfuerzo que supone salir de la zona de confort de la metodología tradicional basada en el libro de texto, cómoda y predecible para optar por la introducción de las TIC en el aula y por la puesta en práctica del currículo bimodal. En este sentido, la actitud del profesor constituye un elemento clave, y la formación, un factor imprescindible para que las innovaciones puedan llevarse a cabo y puedan mejorar la productividad y la eficacia de los procesos de enseñanzaaprendizaje. 


\subsection{Cuando los Resultados Que obTenemos son Malos Quiere decir QUE LO ESTAMOS HACIENDO MAL}

Muchas veces, las quejas más generalizadas del profesorado son que los alumnos no aprenden, no estudian, no progresan, no siguen el ritmo de la clase, pero, sin embargo, no se toman medidas para atajar estos problemas, sino que se sigue haciendo lo mismo en el aula que lo que se hacía hace medio siglo: abrir el libro, leer su contenido y hacer ejercicios más o menos mecánicos, mientras que las pruebas de evaluación siguen basándose en la comprobación de que se ha realizado un aprendizaje memorístico de los contenidos.

Esta metodología ha quedado obsoleta a la luz de la sociedad de la información y del conocimiento que nos rodea. No forma a los alumnos para que puedan desenvolverse en la vida adulta en la que la adquisición de competencias y en concreto de la competencia digital, desempeña un papel clave y además, no consigue motivar al alumnado ni mejorar sus aprendizajes ni sus resultados académicos.

Por lo tanto, es necesario que el profesorado realice una profunda reflexión acerca de cuáles son las competencias y los objetivos del proceso de enseñanza-aprendizaje y de cuál es la metodología más adecuada para su consecución. El papel del profesorado es fundamental para que se opere una necesaria transformación de la enseñanza tradicional en una enseñanza conectada con los intereses y necesidades de la sociedad y del alumnado, dirigida a la adquisición de competencias.

En definitiva, si el profesor no cambia la metodología, no conseguirá que cambien los resultados de sus alumnos.

\section{CONCLUSIONES}

En el contexto actual es necesario reflexionar acerca de cuáles han de ser los objetivos de la educación. Combatir el fracaso y enseñar a los estudiantes a que se desenvuelvan en su dimensión personal, social, académica y profesional han de constituir los principios a partir de los cuales se vertebre la práctica docente. Por ello, hay que tener en cuenta el importante papel de las nuevas tecnologías para que el individuo pueda desarrollarse plenamente, puesto que la comunicación, el conocimiento y el mundo laboral se encuentran muchas veces mediatizados por las TIC. Además, el propio ser humano está sufriendo 
una transformación hacia la i-person, en constante conexión con la información y con los demás usuarios de Internet y la educación no puede permanecer ajena a estos cambios.

Sin embargo, introducir las nuevas tecnologías en el aula es un proceso complejo en el que tienen que intervenir factores como la dotación de material, la organización del centro para su uso y sobre todo, la formación del profesorado en una doble vertiente: competencia digital y competencia didáctica y metodológica de las TIC. Por ello es necesario que el profesorado conozca las ventajas e inconvenientes del uso de las TIC, sus posibilidades organizativas y las distintas herramientas digitales a su alcance. Además, un uso exitoso de las TIC en el aula pasa por la aplicación del currículo bimodal, que aporta un modelo que combina el desarrollo de estrategias de búsqueda, selección, procesamiento, elaboración y presentación de información mediante las TIC con los requisitos mínimos de carácter memorístico que posibilitan la adquisición de los nuevos aprendizajes. Esta nueva perspectiva supone cambios, tanto en la práctica diaria de clase como en la manera de evaluar y de concebir el proceso de aprendizaje, más centrado en el alumno y en la adquisición de habilidades cognitivas y competencias y más restrictivo en las exigencias memorísticas.

En definitiva, el currículo bimodal constituye un enfoque basado en las nuevas tecnologías imbricado con la enseñanza por competencias y en plena consonancia con los principios metodológicos y pedagógicos más actuales, cuyo objetivo fundamental es la mejora de los aprendizajes de los alumnos en el contexto de la sociedad de la información y del conocimiento.

\section{REFERENCIAS BIBLIOGRÁFICAS}

Angrist, J. y Lavy, V. (2002). New evidence on classroom computers and pupil learning. Economic Journal, 112 (482), 735-765.

Attwell, G. (2013). ¿Dónde vamos con los entornos de aprendizaje? En L. Castañeda, y J. Adell (Eds.), Entornos Personales de Aprendizaje: claves para el ecosistema educativo en red (pp. 189-192). Alcoy: Marfil.

Banerjee, A., Cole, S., Duflo, E. y Linden, L. (2007). Remedying education: Evidence from two randomized experiments in India. Quarterly Journal of Economics, 122(3), 1235-1264.

Cabras, S. y Tena, J. (2013). Estimación del efecto causal del uso de ordenadores en los resultados de los estudiantes en la prueba PISA 2012. En PISA 2012, Informe Español, Volumen II: Análisis 
secundario, OCDE-Ministerio de Educación, Cultura y Deporte España.

Castañeda, L. y Adell, J. (2013). La anatomía de los PLEs. En L. Castañeda, y J. Adell (Eds.), Entornos Personales de Aprendizaje: claves para el ecosistema educativo en red (pp. 11-28). Alcoy: Marfil.

Escamilla González, A. (2009). Competencia en el tratamiento de la información y digital. Concepto, componentes, relaciones y recursos. Multiárea. Revista de didáctica, 4, 145-161.

Eurostat. (2014). Early leavers from education and training [en línea] http://ec.europa.eu/eurostat/tgm/table.do?tab=table\&init=1\&languag $\mathrm{e}=$ en\&pcode $=$ tsdsc410\&plugin $=1$ [Consulta: 3 de marzo de 2015].

Fuchs, T. y Woessman, L. (2004). Computers and student learning: Bivariate and multivariate evidence on the availability and use of computers at home and at schools. Brussels Economic Review, 47, 359-389.

Freire, P. (2008). Pedagogía del oprimido. Madrid: S. XXI Editores.

Machin, S., McNally, S. y Silva, O. (2007). New technologies in schools: Is there a payoff? Economic Journal, 117(522), 1145-1167.

Majó, J. y Marquès, P. (2001). La revolución educativa en la era Internet. Barcelona: CissPraxis.

Marquès Graells, P. (2011). Y la evolución humana sigue: ¿eres ya "i-Person"? Tecnonews. [en línea] http://www.tecnonews.info/Y-la-evolucionhumana- sigue--eres-ya--iPerson-/_pE0Aj1BfZN7VtoFZxcYPP8oHseGtVPsPnm-4Gx9fOuJDsPZhDjFS2ou5OiUHcUe [consultado el 3 de marzo de 2015].

Marquès Graells, P. (2013a). ¿Quo vadis escuela? Reflexiones y propuesta para iniciar el camino del cambio (versión 3.0) Chispas y educación [en línea] http://peremarques.blogspot.com.es/2013/08/3-cosas-que-heaprendido-sobre-educacion.html [consultado: 3 de marzo de 2015].

Marquès Graells, P. (2013b). Cap a un nou paradigma educatiu: el currículum bimodal. Aloma: revista de psicologia, ciències de l'educació $i$ de l'esport, 31, 65-72.

Marquès Graells, P. (2014a). Las investigaciones DIM sobre el uso de las pizarras digitales en Educación. Comunicación y pedagogía: Nuevas tecnologías y recursos didácticos, 257-276, 48-51.

Marquès Graells, P. (2014b). ¿Qué han aportado a la mejora educativa las 22 investigaciones DIM? (2004-2014) 2a Parte. DIM, 29 (Suplemento) [en línea] http://es.slideshare.net/peremarques/resultados2-de-las-22investigaciones-dim-2-parte [consultado: 3 de marzo de 2015].

Marquès Graells, P. (2014c, abril). TIC: el objetivo no es innovar, el objetivo es mejorar la formación del alumnado y el éxito escolar. 10 ideas para lograrlo. ¿Por qué nos conviene tener tabletas en el aula y aplicar un 
curriculum bimodal? Ponencia invitada presentada en el IV Congreso Internacional de Competencias Básicas. Competencia digital y tratamiento de la información. Aprender en el siglo XXI, Ciudad Real, España.

Mishra, P. y Koehler, M.J. (2006). Technological Pedagogical Content Knowledge: A Framework for Teacher Knowledge. Teachers College Record, 108 (6), 1017-1054.

Nieto Moreno de Diezmas, E. (2010). Reading comprehension in the context of key competences. Kwartalnik Pedagogiczny, 3 (217), 85-100.

Puentedura, R.R (2014) Learning, Technology and the SAMR Model: Goals, Processes and Practice [en línea] Congreso ISTE, Atlanta 2014 http:/hippasus.com/blog/archives/date/2014/06 [consultado: 3 de marzo de 2015].

Tárraga, R., Sanz-Cervera, P., Pastor, G. y Fernández, M. I. (2014). Herramientas TIC para la intervención educativa en estudiantes con altas capacidades. Un estudio de caso. Revista Didáctica, Innovación y Multimedia, 30 [en línea] http://dim.pangea.org/revista30.htm [consultado: 3 de marzo de 2015]. 